\title{
Space-Time-Color (STC) Scheme with Symbol-Hopping for Color Shift Keying (CSK)Modulation
}

This paper was downloaded from TechRxiv (https://www.techrxiv.org).

\section{LICENSE}

CC BY 4.0

SUBMISSION DATE / POSTED DATE

25-10-2021 / 06-11-2021

\section{CITATION}

Ximenes, Leandro; Arthur, Rangel; Rodrigues, igor Santos Cruz (2021): Space-Time-Color (STC) Scheme with Symbol-Hopping for Color Shift Keying (CSK)Modulation. TechRxiv. Preprint. https://doi.org/10.36227/techrxiv.16869483.v1

DOI 


\title{
Space-Time-Color (STC) Scheme with Symbol-Hopping for Color Shift Keying (CSK) Modulation
}

\author{
Igor S. C. Rodrigues, Leandro R. Ximenes, Member, IEEE, and Rangel Arthur
}

\begin{abstract}
This paper proposes a novel coding scheme for Visible Light Communications (VLC) systems using symbol mapping permutations on the color domain. The permutation is done through symbol-hopping over the points of an optimized 4-CSK constellation. This scheme provides diversity gains, promises robustness against monochromatic channel degradation, and increases the information security of the communication link. It can also be used in conjunction with Single-Input and Single-Output (SISO) systems, as well as in Multiple-Input and Multiple-Output (MIMO) systems. Monte Carlo computational simulations evaluate the performance of the proposed scheme over the conventional QuadLED (QLED) CSK system and other codes, showing superior coding and diversity gains over two direct competitors, under a Rician flat-fading channel.
\end{abstract}

Index Terms-Visible Light Communications, Color Shiftkeying, Symbol permutation, Optical wireless communications

\section{INTRODUCTION}

Visible Light Communications (VLC) is an emerging technology, standardized by IEEE at 2011 [1], that uses the visible light spectrum to transmit data as well as to provide illumination. It can provide higher data rates, cheaper implementation, and greater security than its Radio Frequency (RF) competitors [2].

Color-Shift-Keying (CSK) is a modulation for VLC systems that maps the symbols on different wavelength combinations. This modulation keeps constant power throughout all transmission, avoiding the harmful flickering effect of other intensity modulations [3]. The standard CSK modulation is implemented with three colored Light-Emitting Diodes (LEDs) (TLED/CSK) [1], whereas the enhanced QLED/CSK modulation uses four [4].

Following traditional RF communication systems, recent works brought Space-Time Block Codes (STBC) to improve optical wireless systems [5]-[10]. Paper [5] presents an Alamouti-type STBC for Free-Space Optics (FSO) using OnOff Keying (OOK) and Pulse-Position Modulation (PPM), and [6] makes an extension to this approach to enable more than two transmit LEDs. The work in [7] analyzes Orthogonal Space-Time Block Codes (OSTBC) for high-order intensity

Igor S. C. Rodrigues and Leandro R. Ximenes are with the School of Technology, State University of Campinas (UNICAMP), Brazil, e-mails: i263014@dac.unicamp.br, leandro@ft.unicamp.br, rangel@ft.unicamp.br.

This work has been supported by CNPq-Brazil, under project 427404/20185, and by CAPES-Brazil - Finance code 001

Manuscript received July 13, 2021. modulations in FSO systems, using PPM, Pulse-Width Modulation (PWM), and Amplitude-Shift-Keying (ASK) modulation. In [8], the authors propose an Alamouti-type coding for TLED CSK systems. This last work is not adapted to QLED systems and has restrictive limitations on its design, with a negative impact on the minimum distance in the constellation diagram. In [9] a novel STBC scheme is proposed for MIMOVLC to control the intrinsic dimming and flickering issues of the OOK modulation. The work [10] presents a SpaceTime-Color (STC) block-code for QLED systems to address the monochromatic degradation of the VLC channel, where a propagation scenario with wavelength-selectivity is present.

Other researchers also seek to optimize the CSK constellation design [11]-[14]. The work in [11] presents an optimization method to obtain the largest minimum Euclidean distances possible for different sets of chromaticity and constellation sizes, while [12] optimizes the constellation for the QLED CSK system for a known channel, maximizing the minimum Euclidean distance using an iterative algorithm. Furthermore, the work [13] proposes rotated constellations dedicated to channels subjected to signal-dependent noise. Random constellation rotation is also used in [14] to decrease information leakage without undermining or improving error performances.

This paper presents a novel transmission scheme for the QLED modulation, called Color-Hopping Space-Time (CHST), based on the pseudorandom permutations of the symbols onto the CSK constellation. The proposed space-timecolor coding scheme lightly resembles the Frequency Hopping Spread Spectrum (FHSS), except that the hopping (permutation) is done on the symbol-color mapping. By resorting to a symbol-hopping on an optimized QLED/4-CSK constellation diagram, the proposed scheme retains the optimal average BER of such modulation. Moreover, this scheme can provide higher diversity order than [10], while bringing intrinsic security enhancements as in [14]. It is empirically shown in [10] that a single symbol permutation in the CSK mapping can increase the link robustness against monochromatic channel attenuation, by providing color diversity to the scheme. The generalized permutation scheme presented in this paper allows even greater diversity gains, for any number of arrays of LEDs and Photodiodes (PDs).

This paper brings the concept of the proposed scheme and the matrix-based modeling for SISO and MIMO systems (Section 1), the condition for using a Zero-Forcing (ZF) equalizer for color calibration (Section II), and, finally, numerical 
evaluations prove the validity of the paper's contributions (Section III).

\section{COlOR-Hopping Scheme}

This section presents the proposed transmission scheme, based on the permutation of the QLED/4-CSK symbol mapping. The proposed CHST coding employs a space-timecolor multiplexing approach, in a way that symbols can be allocated to different transmit LEDs with the symbols hopping (permuting) their mappings at the (4-CSK) color diagram at different time-slots.

Firstly, we present a simplified version of the scheme for a single pair of LED-PD arrays (namely SISO case), and then we will generalize the scheme for multiple pairs of arrays (MIMO case).

\section{A. SISO case}

Assume a point-to-point VLC block transmission based on the QLED/4-CSK modulation. At the transmitter, $\mathbf{S} \in \mathbb{R}^{N \times K_{T}}$ is a matrix containing the $N$ symbols to be multiplexed to the $K_{T}$ LEDs of a single transmit array, i.e., for QLED $K_{T}=$ 4. For the QLED/4-CSK modulation, $\mathbf{S}$ is a sparse matrix, containing just one non-zero element per row, i.e., just one LED lit at a time with power $P_{T}$ [4].

For the receiver, a single array of $K_{R}$ photodiodes is used. Ideally, for a case with no color cross-talk, it is expected that $K_{T}=K_{R}$, with each color present on the LED array matching a PD of the same wavelength. In general, the communication model for a QLED/CSK system can be given by

$$
\mathbf{Y}=\mathbf{H}_{e} \mathbf{S}^{T},
$$

where $\mathbf{H}_{e} \in \mathbb{R}^{K_{R} \times K_{T}}$ is the effective channel matrix, $\mathbf{Y} \in$ $\mathbb{R}^{K_{R} \times N}$ are the signals received by the $K_{R}$ PDs for the $N$ transmitted symbols. For the particular case where there is no color cross-talk, the channel matrix $\mathbf{H}$ is a diagonal matrix whose non-null entries represent the attenuation gains for each color.

In the following paragraphs, a Color-Hopping scheme is proposed to improve either diversity or multiplexing gains in QLED/CSK systems. A general SISO model will be valid for both scenarios.

1) Diversity scheme: Let the block of $N$ symbols in $\mathbf{S}$ be repeated $P$ times along the time domain. At each repetition, the symbol mapping upon the color map is permuted in an apparently pseudorandom fashion, although known by both transmitter and receiver. This means that the mapping of CSK symbols on the $K_{T}$ LEDs is shifted according to these permutations. Fig. 1 shows the proposed transmission scheme with four LEDs.

Due to symbol permutation, a new symbol matrix $\mathbf{S}_{p}$ is generated from $\mathbf{S}$, where $p=1, \cdots, P$ indicates the $p$-th permutation. Thus, the received symbol matrix on the $p$-th interval is now redefined from (1) as

$$
\mathbf{Y}_{p} \triangleq \mathbf{H}_{p} \mathbf{S}_{p}^{T}=\mathbf{H}_{p} \mathbf{M}_{p} \mathbf{S}^{T} \in \mathbb{R}^{K_{R} \times N},
$$

where $\mathbf{S}_{p}=\mathbf{S M}_{p}^{T}$, and $\mathbf{M}_{p}$ is a permutation matrix of size $K_{T} \times K_{T}$. Channel matrix $\mathbf{H}_{p} \in \mathbb{R}^{K_{R} \times K_{T}}$ denotes a (possibly) time-variant block-fading channel.

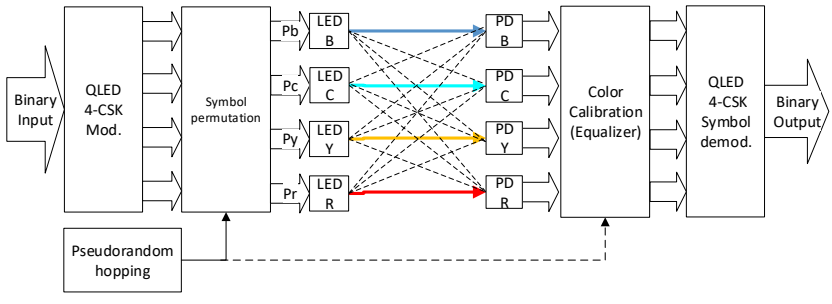

Figure 1: Proposed transmission scheme

Combining the received signals from (2) for all $p=$ $1,2, \cdots, P$, one gets the combined signal matrix $\overline{\mathbf{Y}}$

$$
\begin{aligned}
\overline{\mathbf{Y}} & =\left[\begin{array}{llll}
\mathbf{Y}_{1}^{T} & \mathbf{Y}_{2}^{T} & \cdots & \mathbf{Y}_{P}^{T}
\end{array}\right]^{T}, \\
& =\left[\begin{array}{c}
\mathbf{H}_{1} \mathbf{M}_{1} \\
\vdots \\
\mathbf{H}_{P} \mathbf{M}_{P}
\end{array}\right] \mathbf{S}^{T} \in \mathbb{R}^{K_{R} P \times N} .
\end{aligned}
$$

By comparing (1) to (3), the new effective channel matrix $\mathbf{H}_{e} \in \mathbb{R}^{K_{R} P \times K_{T}}$ can be deducted as

$$
\mathbf{H}_{e}=\operatorname{blkdiag}\left(\mathbf{H}_{1}, \mathbf{H}_{2}, \cdots, \mathbf{H}_{P}\right)\left[\begin{array}{llll}
\mathbf{M}_{1}^{T} & \mathbf{M}_{2}^{T} & \cdots & \mathbf{M}_{P}^{T}
\end{array}\right]^{T},
$$

where blkdiag (.) refers to a diagonal block matrix.

2) Multiplexing scheme: Assume now that in (2) one has that $\mathbf{Y}_{p} \triangleq \mathbf{H}_{p} \mathbf{M}_{p} \mathbf{S}_{p}^{T}$. In other words, at every permutation interval, a new set of independent symbols are permuted and then transmitted through the channel. Vertically stacking $\mathbf{Y}_{p}$ for $p=1,2, \cdots, P$ yields

$$
\overline{\mathbf{Y}}=\left[\begin{array}{c}
\mathbf{H}_{1} \mathbf{M}_{1} \mathbf{S}_{1}^{T} \\
\vdots \\
\mathbf{H}_{P} \mathbf{M}_{P} \mathbf{S}_{P}^{T}
\end{array}\right]=\mathbf{H}_{e} \overline{\mathbf{S}}^{T}
$$

where $\overline{\mathbf{S}} \triangleq\left[\mathbf{S}_{1} \mathbf{S}_{2} \cdots \mathbf{S}_{P}\right] \in \mathbb{R}^{N \times K_{T} P}$. Now, the effective channel (4) is replaced by

$$
\mathbf{H}_{e} \triangleq \overline{\mathbf{H}} \overline{\mathbf{M}}
$$

where

$$
\begin{aligned}
\overline{\mathbf{H}} & =\operatorname{blkdiag}\left(\mathbf{H}_{1}, \mathbf{H}_{2}, \cdots, \mathbf{H}_{P}\right) \in \mathbb{R}^{K_{R} P \times K_{T} P}, \\
\overline{\mathbf{M}} & =\operatorname{blkdiag}\left(\mathbf{M}_{1}, \mathbf{M}_{2}, \cdots, \mathbf{M}_{P}\right) \in \mathbb{Z}^{K_{T} P \times K_{T} P} .
\end{aligned}
$$

3) General SISO model: Note that (7) also appears in (4). Thus, for the SISO model with either (3) or (5), we can generalize the (noiseless) received signals as

$$
\overline{\mathbf{Y}}^{(\mathrm{SISO})}=\overline{\mathbf{H}}^{(\mathrm{SISO})} \overline{\mathbf{M}} \overline{\mathbf{S}}^{T},
$$

where $\overline{\mathbf{H}}^{(\mathrm{SISO})} \triangleq \overline{\mathbf{H}}$ is given by (7), and

$$
\overline{\mathbf{M}}= \begin{cases}{\left[\mathbf{M}_{1}^{T} \cdots \mathbf{M}_{P}^{T}\right]^{T} \in \mathbb{R}^{K_{T} P \times K_{T}},} & \text { if } \mathbf{S}_{i}=\mathbf{S}_{p} \forall i, p, \\ \text { blkdiag }\left(\mathbf{M}_{1}, \cdots, \mathbf{M}_{P}\right) \in \mathbb{R}^{K_{T} P \times K_{T} P}, & \text { otherwise, }\end{cases}
$$

and

$$
\overline{\mathbf{S}}= \begin{cases}\mathbf{S} \in \mathbb{R}^{N \times K_{T}}, & \text { if } \mathbf{S}_{i}=\mathbf{S}_{p} \forall i, p, \\ {\left[\mathbf{S}_{1} \mathbf{S}_{2} \cdots \mathbf{S}_{P}\right] \in \mathbb{R}^{N \times K_{T} P},} & \text { otherwise }\end{cases}
$$


whether the CHST scheme works to achieve improved diversity (i.e., $\mathbf{S}_{i}=\mathbf{S}_{p}$ ) or data rate gain (i.e., $\mathbf{S}_{i} \neq \mathbf{S}_{p}$ ). Note that the original SISO QLED/4-CSK scheme in [4] is similar to our SISO scheme if $P=1, L_{R}=L_{T}=1$, and $\overline{\mathbf{M}}=\mathbf{I}_{K_{T}}$, where $\mathbf{I}_{K_{T}}$ is the identity matrix of size $K_{T} \times K_{T}$. In addition, our ColorHopping scheme is similar to the conventional Repetition Code $(\mathrm{RC})$ if $\mathbf{M}_{p}=\mathbf{I}_{K_{T}} \forall p \in\{1,2, \cdots, P\}$.

\section{B. General MIMO case}

We can generalize (9) to a MIMO system by arranging the received signals of every LED-PD pair, which leads to

$$
\begin{aligned}
\overline{\mathbf{Y}}^{(\text {MIMO })} & =\left[\begin{array}{ccc}
\overline{\mathbf{H}}^{(1,1)} & \cdots & \overline{\mathbf{H}}^{\left(1, L_{T}\right)} \\
\vdots & \ddots & \vdots \\
\overline{\mathbf{H}}^{\left(L_{R}, 1\right)} & \cdots & \overline{\mathbf{H}}^{\left(L_{R}, L_{T}\right)}
\end{array}\right]\left(\mathbf{I}_{L_{T}} \otimes \overline{\mathbf{M}}\right) \overline{\mathbf{S}}^{T}, \\
& =\overline{\mathbf{H}}^{(\mathrm{MIMO})}\left(\mathbf{I}_{L_{T}} \otimes \overline{\mathbf{M}}\right) \overline{\mathbf{S}}^{T},
\end{aligned}
$$

where $\otimes$ is the Kronecker product, and $\overline{\mathbf{H}}^{(\mathrm{MIMO})} \in$ $\mathbb{R}^{K_{R} P L_{R} \times K_{T} P L_{T}}$ is the total effective MIMO channel matrix. The submatrix $\overline{\mathbf{H}}^{\left(l_{R}, l_{T}\right)} \in \mathbb{R}^{K_{R} P \times K_{T} P}$ for $l_{R}=\left\{1,2, \cdots, L_{R}\right\}$ and $l_{T}=\left\{1,2, \cdots, L_{T}\right\}$ is the channel between the $l_{R}$-th PD array and the $l_{T}$-th transmit array. From (7) one has that

$$
\overline{\mathbf{H}}^{\left(l_{R}, l_{T}\right)}=\operatorname{blkdiag}\left(\mathbf{H}_{1}^{\left(l_{R}, l_{T}\right)}, \mathbf{H}_{2}^{\left(l_{R}, l_{T}\right)}, \cdots, \mathbf{H}_{P}^{\left(l_{R}, l_{T}\right)}\right) .
$$

Note that $\overline{\mathbf{M}}$ is still given by (10), and $\mathbf{H}_{p}^{\left(l_{R}, l_{T}\right)} \forall p \in$ $\{1, \cdots, P\}$ is still a block-diagonal matrix if there is no color cross-talk, as in the SISO case.

The symbol matrix for the MIMO case is given by

$$
\overline{\mathbf{S}}=\left[\overline{\mathbf{S}}_{1}, \overline{\mathbf{S}}_{2}, \cdots, \overline{\mathbf{S}}_{L_{T}}\right],
$$

where $\overline{\mathbf{S}}_{l_{T}}$ for $l_{T}=1,2, \cdots, L_{T}$ contain the symbols that are multiplexed to the $l_{T}$-th array of $K_{T}$ LEDs. The dimensions of $\overline{\mathbf{S}}_{l_{T}}$ are given by that of (11).

Realize in (12) that the symbol permutation is indeed done on the color domain, not on the spatial domain, i.e., $\overline{\mathbf{M}}$ is independent of the transmit or receive arrays.

\section{SYMBOL DETECTION}

Suppose perfect knowledge of the Channel State Information (CSI) by the receiver for color calibration purposes. If $\overline{\mathbf{M}}$ is also known by it, then it can apply an equalizer to estimate $\overline{\mathbf{S}}$ from (12).

Equalization may be performed by a Zero-Forcing (ZF) equalizer as

$$
\hat{\mathbf{S}}=\left(\mathbf{H}_{e}^{\dagger} \tilde{\mathbf{Y}}\right)^{T}
$$

where $\hat{\mathbf{S}}$ is the estimate of $\overline{\mathbf{S}}$, and $\tilde{\mathbf{Y}}$ is the noisy version of either $\overline{\mathbf{Y}}^{(\mathrm{SISO})}$ or $\overline{\mathbf{Y}}^{(\mathrm{MIMO})}$. The operator $\dagger$ indicates the MoorePenrose inverse.

\section{A. Condition on $L_{R}$ and $L_{T}$ for a $Z F$ equalizer}

As long as the color cross-talk is predicted, the diversity provided by the symbol permutation aids the $\mathrm{ZF}$ equalizer to eliminate this interference, even if it is caused by a lower number of PD arrays $L_{R}$ than the number of LED arrays $L_{T}$.

In (15) the matrix $\mathbf{H}_{e}^{\dagger}$ exists if and only if $\mathbf{H}_{e}$ has fullcolumn rank. As in (12) $\mathbf{H}_{e}=\overline{\mathbf{H}}^{(\mathrm{MIMO})}\left(\mathbf{I}_{L_{T}} \otimes \overline{\mathbf{M}}\right)$, and $\left(\mathbf{I}_{L_{T}} \otimes \overline{\mathbf{M}}\right)$ has always full column-rank, the Proposition 1 can be used to assure that $\mathbf{H}_{e}$ has full column-rank.

Proposition 1. Let a CSK-VLC system implement the permutation-based transmission scheme proposed in this paper, with $\left\{K_{T}, L_{T}, L_{R}, P\right\} \geq 1$. If the diagonal coefficients of $\mathbf{H}_{p}^{\left(l_{R}, l_{T}\right)} \forall\left(p, l_{R}, l_{T}\right)$ are independently drawn from a continuous Gaussian distribution, then with probability close to one the symbol recovery can be carried out by the $Z F$ process in (15) if

$$
K_{R} L_{R} \geq K_{T} L_{T}
$$

Proof. The matrix $\mathbf{H}_{e}$ is left-invertible if and only if it has full column-rank. This is a necessary and sufficient condition. Besides, $\mathbf{H}_{e}$ has full column-rank if and only if its rank is equal to its number of columns, i.e., depending on $\overline{\mathbf{M}}$ in (10)

$$
\operatorname{rank}\left(\mathbf{H}_{e}\right)= \begin{cases}K_{T} L_{T}, & \text { if } \mathbf{S}_{i}=\mathbf{S}_{p} \forall i, p, \\ K_{T} L_{T} P, & \text { otherwise. }\end{cases}
$$

For the MIMO scenario, one has that $\mathbf{H}_{e}=$ $\overline{\mathbf{H}}^{(\mathrm{MIMO})}\left(\mathbf{I}_{L_{T}} \otimes \overline{\mathbf{M}}\right)$. Since from Sylvester's rank inequality

$$
\operatorname{rank}\left(\mathbf{H}_{e}\right) \geq \operatorname{rank}\left(\overline{\mathbf{H}}^{(\mathrm{MIMO})}\right)+\operatorname{rank}\left(\mathbf{I}_{L_{T}} \otimes \overline{\mathbf{M}}\right)-L_{T} K_{T} P,
$$

then $\mathbf{H}_{e}$ is full column-rank by (17) if

$$
\begin{array}{r}
\operatorname{rank}\left(\overline{\mathbf{H}}^{\text {(MIMO) })+\operatorname{rank}\left(\mathbf{I}_{L_{T}} \otimes \overline{\mathbf{M}}\right)-L_{T} K_{T} P}\right. \\
= \begin{cases}K_{T} L_{T}, & \text { if } \mathbf{S}_{i}=\mathbf{S}_{p} \forall i, p, \\
K_{T} L_{T} P, & \text { otherwise. }\end{cases}
\end{array}
$$

If the channel submatrix $\mathbf{H}_{p}^{\left(l_{R}, l_{T}\right)} \forall\left(p, l_{R}, l_{T}\right)$ has independent and normally distributed diagonal elements by the statement of Proposition 1, then one has with probability close to one that $\overline{\mathbf{H}}^{\left(l_{R}, l_{T}\right)}$ in (13) is full rank. Moreover, any two channel submatrices are uncorrelated. By extension, $\overline{\mathbf{H}}^{(\mathrm{MIMO})}$ is also a full rank matrix, i.e.,

$$
\operatorname{rank}\left(\overline{\mathbf{H}}^{(\mathrm{MIMO})}\right)=\min \left(K_{R} P L_{R}, K_{T} P L_{T}\right) .
$$

Moreover, as $\mathbf{M}_{\underline{p}}$ is a full rank square matrix for all values of $p$, then $\left(\mathbf{I}_{L_{T}} \otimes \mathbf{M}\right)$ is full column-rank, which yields

$$
\operatorname{rank}\left(\mathbf{I}_{L_{T}} \otimes \overline{\mathbf{M}}\right)= \begin{cases}L_{T} K_{T}, & \text { if } \mathbf{S}_{i}=\mathbf{S}_{p} \\ L_{T} K_{T} P, & \text { otherwise. }\end{cases}
$$

Combining (19) and (20) in (18), then (17) is always satisfied if

$$
\min \left(K_{R} P L_{R}, K_{T} P L_{T}\right)=K_{T} L_{T} P .
$$

With $\left\{K_{T}, L_{T}, L_{R}, P\right\} \geq 1$, then (21) it is satisfied if $K_{R} P L_{R} \geq K_{T} P L_{T}$, which leads to $K_{R} L_{R} \geq K_{T} L_{T}$, ending this proof. 


\section{Simulation RESUlts}

In this section, the Bit Error Rate (BER) performances of the CHST scheme are validated through simulation comparisons between the proposed scheme and some of its competitors, i.e., the conventional QLED/CSK scheme [4], the conventional Repetition Coding (RC) and the Constant Weight Space-Time Coding (CWSTC) scheme [9].

A two-tap channel model is used to simulate an indoor propagation scenario where the communication takes place. More specifically, both transmit and receive arrays are positioned at the center of a square room of $36 \mathrm{~m}^{2}$, respectively on the ceiling and the floor, while the room is $4 \mathrm{~m}$ in height. The first channel tap represents the line-of-sight component, with an average $-36.12 \mathrm{~dB}$ power gain and $9.42 \mathrm{~ns}$ of propagation delay. The second tap represents the non-line-of-sight scattered paths with only a single-bounce on the walls, with a -65.83 $\mathrm{dB}$ gain and $26.97 \mathrm{~ns}$ delay. In these simulations, the receiver has perfect channel knowledge for color calibration purposes. Doppler spread is neglected, and all the channels are flatfading.

BER is analyzed with Monte Carlo simulations, with channel coefficients being sorted out at every new block of $N K_{T}$ symbols. In total, $10^{6} 4-\mathrm{CSK}$ symbols are transmitted per simulated $E_{b} / N_{o}$ value, where $E_{b}$ is the bit energy (W.s), and $N_{o}$ is the (additive white Gaussian) noise spectral density $(\mathrm{W} / \mathrm{Hz})$. In addition, all transmit and receive arrays are considered uncorrelated, in the sense that every non-null channel coefficient is an independent and identically distributed (i.i.d.) Gaussian variable. We also assume that there is no color crosstalk, with $K_{R}=K_{T}=4$.

Fig. 2 compares the proposed CHST scheme implemented on a SISO sytem with the conventional RC and the Constant Weight Space-Time Code (CWSTC) [9]. This last scheme has many qualities that make it a proper competitor to ours: the CWSTC scheme employs a block space-time coding for MIMO-VLC communications, where a code design was proposed to attend dimming requirements and avoid flickering. In our proposed Space-Time-Color MIMO-VLC scheme, dimming and flickering are inherently dealt with by the CSK modulation.

For the CWSTC scheme, its spectral efficiency (in bps/Hz) is given by $s=\log _{2}\left(N_{C W}\right)$ [9], where $N_{C W}$ represents the number of white LEDs and PDs as well as the number of OOK symbols multiplexed to each LED. Both RC and our proposed scheme have spectral efficiency given by $s=2 N L_{T} / N P=$ $2 L_{T} / P$, as $2 N$ bits (i.e., $N$ 4-CSK symbols) are transmitted during $N P$ symbol periods. In Fig. $2, L_{T}=1$, so $s=2 / P$. The spectral efficiency of the conventional QLED/4-CSK scheme, with no repetition or permutation, is equal to $s=2$.

For the same spectral efficiency $s=2$, the CWSTC scheme outperforms the conventional QLED scheme by around $2 \mathrm{~dB}$. However, for $s=1$, both RC and the CHST schemes are better than CWSTC. In the scenario where the channel is timeinvariant during symbol repetitions, i.e., $\mathbf{H}_{1}^{(1,1)}=\mathbf{H}_{2}^{(1,1)}=\ldots=$ $\mathbf{H}_{P}^{(1,1)}$, the proposed CHST scheme presents an approximately $6 \mathrm{~dB}$ coding gain over both RC and CWSTC for a target BER of $10^{-4}$. For the scenario where $\mathbf{H}_{1}^{(1,1)} \neq \mathbf{H}_{2}^{(1,1)} \neq \ldots \neq \mathbf{H}_{P}^{(1,1)}$, the RC and the CHST schemes present the same performance, both with a diversity gain over the CWSTC system.

Although it is clear that diversity gains are achieved thanks to the channel variation, the coding gains for the time-invariant channel justify the use of permutation instead of the simple symbol repetition. It can also be reasonable to assume that CHST is more robust to monochromatic channel degradation than the RC scheme, as [10] has shown that even a single color hopping can be beneficial to cope with this propagation issue.

The fact that the proposed scheme can have better control of the multiplexing and diversity gains by varying $L_{T}$ or $P$ is another differential in relation to CWSTC. The minimum spectral efficiency $s=1$ for CWSTC brings its best possible BER performance, as shown in Fig. 2, while the spectral efficiency of CHST could be even more sacrificed to improve error performance. Finally, due to the pseudorandom permutations, the CHST scheme provides additional information security over its competitors.

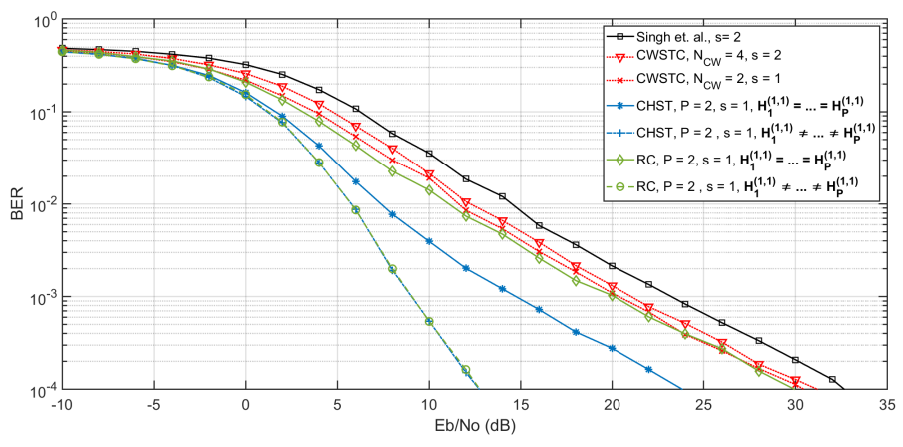

Figure 2: BER comparison for different spectral efficiency $s$.

Fig. 3 presents the BER comparison for a different number of permutation blocks $P$ and of receive arrays $L_{R}$. Here there is only one transmit array $\left(L_{T}=1\right)$, which leads to a coding rate of $r=1 / P$. For all curves in Fig. 3, the channels are considered time-invariant during the symbol permutations. For the SISO scenario (i.e., $L_{R}=1$ ) in Fig. 3, the proposed CHST scheme presents a diversity gain over the RC and Singh et. al. [4] schemes for every $P>2$, while for $P=2$ only a coding gain is visible. By allowing an increase in the number of permutations, the proposed scheme reduces probability of repeating the same color-symbol mapping, explaining the visible diversity gain over RC.

However, there are limited diversity gains for higher values of $P$ (e.g. $P=8$ ). From the simple SISO case in (3), one can deduce that, if the channel is invariant to $p$, then the maximum number of random permutation matrices that can generate diversity in the channel depends on the size of the CSK alphabet, i.e., $P=4$ for $4-C S K$. Consequently, implementing the scheme for a higher-order of modulation may allow even greater diversity gains. It is important to notice that since there is no space diversity technique present (i.e., SISO case) and there is no conventional time diversity (i.e., time-invariant channels), the diversity gain for $P>2$ stems from the color diversity (i.e., symbol-color hopping). 
Still for the SISO case, Fig. 3 also shows that for a target BER of $10^{-4}$, the CHST scheme outperforms the RC at high $E_{B} / N_{o}$, with gains of approximately $6 \mathrm{~dB}$ (at $P=2$ ), $16 \mathrm{~dB}$ (at $P=4$ ), and $20 \mathrm{~dB}$ (at $P=8$ ) over its competitor for a target BER of $10^{-4}$. Comparing the SISO case and the SIMO case (i.e., $L_{R}=2$ ), one can see that the proposed symbol hopping scheme allows obtaining diversity gains without increasing the number of PD arrays, probably a possible positive impact on the cost of the system implementation.

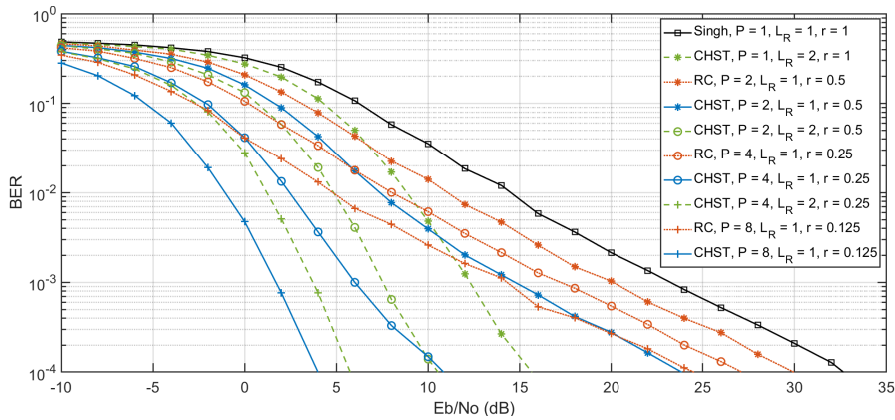

Figure 3: BER comparison for different coding rate $r$.

\section{CONCLUSION}

In this paper, we proposed a novel space-time-color transmission scheme, called Color-Hopping Space-Time (CHST), based on the pseudorandom permutations of the information symbols on the CSK constellation diagram. These permutations, performed on the symbol mapping over the color domain, can be used to increase the diversity order while providing additional information security. Computational evaluations show coding and diversity gains with the CHST scheme over direct competitors.

The observed good BER performances, even at very low SNR values, indicate that the proposed scheme may be used in scenarios where there is critical obstruction of the line of sight, as the receiver may be in a shadowed area. In short, the CHST scheme can be implemented to achieve a good tradeoff between a given BER target, overall data rate, and costefficiency, especially if the increase of PD arrays is restricted.

\section{REFERENCES}

[1] IEEE Standard for local and metropolitan area networks-Part 15.7: Short-Range Optical Wireless Communications," in IEEE Std 802.15.72018 (Revision of IEEE Std 802.15.7-2011), vol., no., pp.1-407, 23 April 2019, doi: 10.1109/IEEESTD.2019.8697198.

[2] D. C. O'Brien, L. Zeng, H. Le-Minh, G. Faulkner, J. W. Walewski and S. Randel, "Visible light communications: challenges and possibilities," 2008 IEEE 19th Int. Symp. on Pers., Indoor and Mobile Radio Commun., Cannes, 2008, pp. 1-5, doi: 10.1109/PIMRC.2008.4699964.

[3] S. Rajagopal, R. D. Roberts and S. Lim, "IEEE 802.15.7 visible light commun.: modulation schemes and dimming support," in IEEE Commun. Mag., vol. 50, no. 3, pp. 72-82, March 2012, doi: 10.1109/MCOM.2012.6163585.

[4] R. Singh, T. O'Farrell and J. P. R. David, "An enhanced color shift keying modulation scheme for high-speed wireless visible light communications," in J. of Lightw. Technol., vol. 32, no. 14, pp. 2582-2592, 15 July 15, 2014, doi: 10.1109/JLT.2014.2328866.

[5] M. K. Simon and V. A. Vilnrotter, "Alamouti-type space-time coding for free-space optical communications with direct detection," in IEEE Trans. on Wireless Commun., vol. 4, no. 1, pp. 35-39, Jan. 2005, doi: 10.1109/TWC.2004.840216.
[6] V. Khare and D. Chandra, "Extended Alamouti space time coding scheme with Turbo Coding for free space Optical communications," 2011 Int. Conf. on Comput. Intell. and Commun, Netw., Gwalior, 2011, pp. 359-362, doi: 10.1109/CICN.2011.76.

[7] T. Ren, C. Yuen, Y. L. Guan and G. Tang, "High-order intensity modulations for OSTBC in free-space Optical MIMO communications," in IEEE Wireless Commun. Lett., vol. 2, no. 6, pp. 607-610, December 2013, doi: 10.1109/WCL.2013.081513.130360.

[8] L. Jia, J. Wang, J. Wang and M. Chen, "Comparison of different space time coding for transmit diversity in VLC syst. with CSK modulation," 2015 IEEE Int. Broadband and Photon. Conf. (IBP), Bali, 2015, pp. 40-48, doi: 10.1109/IBP.2015.7230763.

[9] J. -N. Guo, J. Zhang, Y. -Y. Zhang, G. Xin and L. Li, "Constant Weight Space-Time Codes for Dimmable MIMO-VLC Systems," in IEEE Photonics Journal, vol. 12, no. 6, pp. 1-15, Dec. 2020, Art no. 7906815, doi: 10.1109/JPHOT.2020.3036648.

[10] I. S. Cruz Rodrigues and L. R. Ximenes, "Space-time-color coding for QuadLED color shift keying modulation for visible light communications," 2020 IEEE Latin-American Conf. on Commun. (LATINCOM), Santo Domingo, Dominican Republic, 2020, pp. 1-6, doi: 10.1109/LATINCOM50620.2020.9282313.

[11] A. E. Aziz, K. T. Wong and J. Chen, "Color-shift keying - how its largest obtainable "minimum distance" depends on its preset operating chromaticity and constellation size," in J. of Lightw. Technol., vol. 35, no. 13, pp. 2724-2733, 1 July1, 2017, doi: 10.1109/JLT.2017.2693363.

[12] X. Liang, M. Yuan, J. Wang, Z. Ding, M. Jiang and C. Zhao, "Constellation design enhancement for color-shift keying modulation of Quadrichromatic LEDs in visible light Communications," in J. of Lightw. Technol., vol. 35, no. 17, pp. 3650-3663, 1 Sept.1, 2017, doi: 10.1109/JLT.2017.2720579.

[13] Q. Gao, K. Qaraqe and E. Serpedin, "Rotated color shift keying for visible light communications with signal-dependent noise," in IEEE Commun. Lett., vol. 24, no. 4, pp. 844-848, April 2020, doi: 10.1109/LCOMM.2020.2967377.

[14] H. Lu, L. Zhang and X. Liu, "High-security colour shift keying modulation scheme with chaos-based constellation rotation for VLC systems," 2016 10th IEEE Int. Conf. on Anti-counterfeiting, Secur., and Identification (ASID), 2016, pp. 20-24, doi: 10.1109/ICASID.2016.7873889. 